\title{
EXECUTIVE FUNCTIONS DEFICIT IN MILD COGNITIVE IMPAIRMENT
}

Latchezar Traykov, MD, PhD, DSci, ${ }^{\mathrm{a}, \mathrm{b}}$ Nadine Raoux ${ }^{\mathrm{c}}$, Florence Latour, MD, ${ }^{\mathrm{d}}$ Livia

Gallo, MD ${ }^{\mathrm{a}, \mathrm{e}}$ Olivier Hanon, MD, PhD, ${ }^{\mathrm{d}}$ Sophie Baudic, $\mathrm{PhD},{ }^{\mathrm{f}}$ Catherine Bayle, MD, ${ }^{\mathrm{d}}$

Emilie Wenisch, MD, ${ }^{\mathrm{d}}$ Philippe Remy, MD, PhD, ${ }^{\mathrm{a}}$ Anne-Sophie Rigaud, MD, PhD, ${ }^{\mathrm{d}}$

${ }^{\text {a} H o ̂ p i t a l ~ H e n r i ~ M o n d o r, ~ U n i v e r s i t y ~ P a r i s ~ X I I, ~ C r e ́ t e i l, ~ F r a n c e ~}$

${ }^{\mathrm{b}}$ Medical University, Department of Neurology, Sofia, Bulgaria

${ }^{c}$ INSERM Unit 330, Bordeaux, France

${ }^{\mathrm{d}}$ Hôpital Broca, Paris, France

${ }^{\mathrm{e}}$ Department of Neurosciences, University of Padova, Italy

fINSERM E-332, CHU Ambroise Paré, Boulogne-Billancourt, France

\section{Corresponding author:}

Prof. Latchezar Traykov, MD, PhD, DSci

Medical University

University Hospital Alexandrovska

Department of Neurology

1, St. Georgi Sofiiski str

1431 Sofia, Bulgaria

Tel/Fax: (359-2) 9526787

e-mail: traykov_1@yahoo.fr

Running title: Executive functions deficit in MCI 
Key words: Mild Cognitive Impairment; Neuropsychology; Attention; Executive functions; Memory; Alzheimer's disease. 


\begin{abstract}
Objective: To investigate whether patients diagnosed as amnestic mild cognitive impairment (MCI) have also impairment in attention/executive functions and, therefore to clarify whether all subcomponents of executive control are equally affected in MCI.

Background: MCI refers to the transitional state between normal ageing and dementia. Amnestic MCI is characterized by impaired episodic memory, although subtle impairment of executive functions has been noted on neuropsychological tests.

Methods: We investigated $20 \mathrm{MCI}$ patients and 20 normal controls using episodic memory, attention/executive functions, language and praxis tests.

Results: MCI patients had a significantly lower scores on all measures of the Free and Cued Selective Reminding Test $(\mathrm{p}<0.05$ to 0.01$)$ than controls. Furthermore MCI had a greater number of perseverations $(\mathrm{p}<0.01)$ on Modified Card Sorting Test and the lowest performance on the Stroop test $(\mathrm{p}<0.02)$.

Conclusion: Our findings showed impairment in episodic memory performance in MCI as compared to that of controls. In addition, MCI patients had problems with response inhibition, switching and cognitive flexibility which encompass various aspects of executive functions. This suggests that MCI may be identified by using a more detailed procedure for the assessment of cognitive decline than the evaluation of memory alone.
\end{abstract}




\section{Introduction}

The recent developments in drug treatments for Alzheimer's disease (AD) have highlighted the importance of early diagnosis and the need to characterize the cognitive profile of the earliest stages of the disease.

Recent research has identified a transitional state between the cognitive changes of normal aging and $\mathrm{AD}$, known as mild cognitive impairment (MCI). ${ }^{1,2}$ Patients with MCI have a memory impairment that is higher than that expected for their age, yet they do not meet commonly accepted criteria for dementia or AD because their cognitive deficits are limited to memory alone and their everyday abilities are preserved. Although MCI can present a variety of symptoms, when memory loss is the predominant symptom it is termed "amnestic MCI" and the risk is higher for the development of $\mathrm{AD}{ }^{2}$ These patients progress to $\mathrm{AD}$ at a rate of $10 \%$ to $15 \%$ per year, as compared to elderly individuals without MCI, who convert at rates of $1 \%$ to $2 \% .{ }^{1}$ However, subsequent work has also indicated that $\mathrm{MCI}$ is heterogeneous in its clinical presentation and should be considered in a broad clinical context. ${ }^{3}$ The principal cognitive impairment can be amnestic, single nonmemory domain or involving multiple cognitive domains (with or without a memory impairment).

Several studies have suggested that an impairment of memory is most common in amnestic $\mathrm{MCI}$ and is the fundamental aspect of cognition to evaluate. ${ }^{4}$ Petersen et al. ${ }^{1}$ reported verbal episodic memory performance in an MCI group that was as impaired as that seen in mild AD. However, the same MCI group's performance on measures assessing other cognitive domains (naming, executive functions, etc.) was equivalent to that of healthy older controls. 
Other studies of MCI report cognitive deficits similar to those described by Petersen and colleagues. $^{5,6}$

However, in recent years, the literature has reported that, while memory is the hallmark of patients with amnestic MCI, they are impaired on a variety of tasks that have commonly been considered a measure of executive functions. ${ }^{7}$ Executive functions are encompassing a number of cognitive abilities which generally have been conceptualized as controlling or guiding behavior in a top-down fashion such as decision-making, planning, self monitoring, and behavior initiation, organization and inhibition. ${ }^{8}$ Within this context Ready et al. ${ }^{9}$ demonstrated that increased executive impairment is common in patients with amnestic $\mathrm{MCI}$ and is evident in patients who are not functionally impaired. Similarly, Perry et al. ${ }^{10}$ reported particular problems with response inhibition and attentional switching in a group of patients who were only impaired on episodic memory tests. Finally, Rapp et al. ${ }^{11}$ reported recently that tasks requiring executive control are significantly affected in the preclinical phase of $\mathrm{AD}$ and are reliable predictors of the disease before diagnosis. The results of these studies emphasised the importance of attention and executive function in MCI patients. However, it must be pointed out that variability across studies in both tasks used to examine aspects of executive function, and in the MCI definition, makes it difficult to determine which aspects of executive control are affected in MCI. Consequently, we selected executive tasks more "universally" accepted like those mentioned in the abovecited studies. The objective was to avoid to further clouding the issues by introducing additional measures. 
The aims of our study were to investigate whether patients diagnosed as amnestic MCI have also impairment in attention/executive functions and, therefore to clarify whether all subcomponents of executive control are equally affected in MCI.

\section{Materials and methods}

\section{Participants}

Subjects were selected among patients who sought consultation at the Broca Geriatric Day Care Hospital in Paris, between January 1999 and December 1999, because of memory problems or other symptoms of cognitive deterioration. The evaluation procedure consisted of detailed medical history, physical and neurological examinations, psychiatric and cognitive evaluations, laboratory tests, and brain computed tomography (CT) and/or magnetic resonance imaging (MRI). The Clinical Dementia Rating scale (CDR) ${ }^{12}$ was also completed. The psychiatric evaluation included a semistructured interview and the Geriatric Depression Scale (GDS). ${ }^{13}$ Cognitive status was evaluated by the Mini Mental State Examination (MMSE) $)^{13}$ and a screening neuropsychological battery previously described. ${ }^{15,16}$ Laboratory workup included complete blood count and differential, serum electrolytes and glucose, liver and renal function tests, thyroid function tests, VDRL, serum B12 and folate levels. All available information was evaluated by an experienced geriatrician, neurologist (who is also trained in brain imaging), and neuropsychologist. Data from patients with coexisting dementia and major depression (DSM-IV) ${ }^{17}$ were not used. Subjects were included in the study after classification into MCI or NC diagnostic categories. 
MCI group: Twenty consecutive elderly subjects with cognitive deficit failed to meet DSM$\mathrm{IV}^{17}$ criteria for Dementia because the mild cognitive impairment did not interfere significantly with their daily activities. The criteria utilized for the selection of the MCI patients were those proposed by the Mayo Clinic Alzheimer's Disease Center). ${ }^{18}$ The 20 patients in this group had: 1) memory complaint by the patient or a reliable informant, 2) normal global cognitive function (MMSE $>26$ ), 3) objective memory impairment as demonstrated by scores of more than 1.5 SDs below age- and education-matched norms, as previously developed), ${ }^{19} 4$ ) generally preserved functional capacity and activities of daily living both by history and by functional scale $(\mathrm{CDR}=0.5)$ assessment and 5$)$ no evidence of any neurological (verified with CT or MRI ( 6 and 14 respectively), psychiatric, or medical disorder nor of drug use that could induce cognitive deterioration. To avoid subcortical vascular lesions we excluded from the study patients that presented any of the following: history of cardiac disease or minor stroke events; clinical course with abrupt deterioration of cognitive function, stepwise decline, or fluctuation; and evidence of relevant CVD by brain imaging (CT or MRI findings of single or multiple small subcortical infarcts and extensive white matter changes). In addition, patients with coexisting cognitive impairment and major depression (DSM IV criteria) ${ }^{17}$ were excluded from the study.

Normal controls group: To compare the neuropsychological performances we composed a control group of 20 elderly subjects without history or symptoms of psychiatric or neurological disease and with integrity of their cognitive functions. However they had no abnormalities on in depth clinical, neurological, psychiatric and neuropsychological 
examinations. All of the controls underwent brain CT. They were matched to patient groups according to age, sex and educational level.
Deleted: to exclude possible

misclassification

Deleted:

Formatted

\section{Neuropsychological Assessment}

Cognitive functions were evaluated in all participants by a comprehensive neuropsychological battery consisting of subtests and modified short forms of commonly used neuropsychological measures. The neuropsychological assessment was blinded to the clinical diagnosis. Short-term memory was examined by the Digit Span (forward) of the Wechsler Adult Intelligence Scale (WAIS) ${ }^{19}$ and the Corsi Block Tapping Test. ${ }^{20}$ Episodic long-term memory was assessed with the Buschke Free and Cued Selective Reminding Test (FCSRT), ${ }^{21}$ assessing free recall (number of items retrieved over three learning trials), total recall (number of words recalled with free and cued procedures over three learning trials), recognition, and the delayed free and total recall. ${ }^{22}$ Attention and executive functions were tested by the Bells test $)^{23}$ (assessing the time to cross out all bells), the Digit Symbol test, ${ }^{19}$ assessing the number of symbols correctly drew for 90 seconds, the Trail Making Test part A (TMT-A) and part B (TMT-B) ${ }^{24}$ (assessing the time to correctly rely all items in each of the trials), the Modified Card Sorting Test (MCST) ${ }^{25}$ (assessing number of categories achieved and perseverative errors), the Stroop Color Interference Test (SCIT) ${ }^{26}$ (assessing, in the third part, the number of items correctly named in 45 seconds,). Language abilities were assessed by the Fifteen Item Subset of Boston Naming Test (BNT), ${ }^{27}$ the semantic Verbal Fluency ${ }^{28}$ (categories animals, assessing number of category exemplars produced in 60 seconds) and the phonemic Verbal Fluency ${ }^{28}$ (letters F, assessing 
number of words produced with each letter in 60 seconds). Visuospatial abilities were evaluated by the constructional praxis (copy five complex designs). ${ }^{29}$

\section{Statistical Analysis}

Statistical analyses were performed with the SPSS 7.5 software package. We performed multivariate analysis of variance (MANOVA) on the dependent variables of each test with the group factor to eliminate variations that cannot be attributed to the cognitive items under study. An analysis of variance (ANOVA) on each test variable was performed separately.

\section{Results}

Demographic characteristics of the patients and controls were presented in table 1 . The ANOVA was used to examine group differences in age and education scores. There were no significant differences between the patients and controls on these variables. There were also no significant differences on gender $(\chi 2=0.5 ; p=0.46)$. With regard to MMSE, the ANOVA showed that MCI patients differed significantly from normal controls.

Table 1 here 
The neuropsychological performance of the two groups included in this study is shown in Table 2. Multivariate analysis of variance revealed a significant difference between groups on neuropsychological tests, Wilks lambda $=0.203, \mathrm{~F}=3,368, \mathrm{p}<0,006$.

With regard to memory tasks, the ANOVA showed that MCI patients differed significantly from normal controls on all the measures of the FCSRT ( $p<0.05$ to 0.0001 ; see table 2 for F values). Short-term memory, as measured by the forward Digit Span and the Corsi Block Tapping test, was not impaired in the MCI group relative to controls.

Table 2 here

In terms of tasks which encompass various aspects of attention and executive functions, ANOVA revealed that performance on the Stroop test and MCST, was significantly impaired in MCI group relative to controls. However, no significant difference was found between patients and controls on Bells test, Digit symbol test, Trail Making test A and B.

With regard to language tasks, intergroup comparisons indicated that patients performed within normal limits on the category and phonemic Verbal Fluency test and the BNT. Similarly, no significant difference was found between patients and controls on Constructional Praxis as measured by the copy of complex designs. 


\section{Discussion}

Our findings show that in addition to the impairment in the anterograde verbal episodic memory performance MCI patients had problems with response inhibition, switching, cognitive flexibility and abstract thinking which encompass various aspects of executive function. The performance on measures assessing all other cognitive domains (short-term memory, language abilities, constructional praxis, etc.) was comparable to that of normal controls.

Despite this was not the main goal of our study, we would like to stress on the benefits of the Buschke's FCSR test ${ }^{21}$ usage, in spite of the studies that examined memory functioning in MCI using free recall measures. ${ }^{1,2}$ Our findings show that MCI patients recalled significantly fewer words on immediate and delayed free recall than did matched control participants and show, in addition, lesser efficacy of cued recall. Similar results were obtained by Grober et al. ${ }^{30}$ who investigated learning and retention in participants who later developed AD. Considered together, our findings with those of Grober et al. ${ }^{30}$ indicate that detection of MCI and very early AD may be best accomplished by using robust learning tests that control cognitive processing.

In addition to the severely abnormal episodic memory performance, we found a significant executive functions deficit in the MCI group as assessed by the Stroop test and the Modified WCST (number of errors and perseverations). Both tests are well known and validated executive tasks measuring the ability to inhibit irrelevant responses, the set 
shifting and cognitive flexibility. These findings are in agreement with the results of Nagahama et al. ${ }^{31}$ who reported more frequent perseverative errors by the MCI patients on modified WCST and argued that this reflects an aspect of executive dysfunction in MCI.

Our results also support the suggestion of Perry et al, ${ }^{10}$ that some subcomponents of executive control, such as the selective attention and response inhibition may be particularly sensitive to the effect of MCI, while other attentional/executive processes may be relatively preserved. In fact, our patients showed particularly impaired performance on tasks requiring resolution of competing response tendencies and target discrimination with relatively preserved performance on tasks of sustained and divided attention. Like Perry et al, ${ }^{10}$ our study has shown that tasks of selective attention such as the Stroop Test are the most sensitive for picking up attention deficits in MCI or very mild AD patients.

Some authors suggest that executive deficits appear early in $\mathrm{AD}$ but the initial stage of the disease, known as MCI is characterised by amnesia alone with profound loss of episodic memory, despite intact executive functions. ${ }^{10}$ Longitudinal studies employing neuropsychological assessment in small groups of subjects have suggested that, following the amnesia-only phase, deficits in executive functions and/or semantic memory arise before all domains of cognitive processing become affected. ${ }^{32}$ Other studies have shown both episodic memory and executive function tasks to be predictive of latter onset of $\mathrm{AD},{ }^{33}$ and some,${ }^{34}$ indeed report executive functions to be equally predictive for the latter development of AD. Thus, although an episodic memory deficit is generally considered to be the first sign of $\mathrm{AD}$, these longitudinal results support a multiple pattern of deterioration prior to AD. Similarly, recent studies show, that when the clinical syndrome of MCI evolves on a neurodegenerative basis, the multiple-domain type of MCI has a less favorable prognosis than the amnestic type and may represent a more advanced prodromal stage of dementia. ${ }^{35,36}$ Our results of profound memory impairment in association with executive functions deficit in "amnestic MCI" suggest that more extensive examination of executive functions may help the early identification of incipient $\mathrm{AD}_{\text {, }}$ 
Conversely to some previous studies ${ }^{37,38}$ we did not find significant difference between

MCI and NC on verbal fluency test, whereas MCI patients were expected to perform poorer

at least on the semantic fluency task. Murphy et $\mathrm{al}^{38}$ sugested that the reduced efficiency in

accessing semantic fluency, indicates that the early neuropathology of AD may extend

beyond the hippocampus to include cortical areas supporting semantic memory. The most

likely explanation for the spearing of performance of fluency tasks in our MCI patients

concerns the difference of the overall cognitive impairment (MMS score) between our

patients and those of others. Our hypothesis is that our patients' semantic memory is still

intact because of the earlier stage of impairment. Supporting our suggestion are the normal

results of the BNT.

While it is generally agreed that early memory impairment in MCI is associated with entorhinal and medial temporal lesions early in the course of the disease,${ }^{37}$ it is very difficult to explain the early impairment of executive functions in terms of direct early involvement of frontal regions. It must be stressed, however, that the precise anatomical correlates of executive functions are still a matter of debate. Several authors, ${ }^{38}$ have proposed that early disconnection of the cortico-cortical tracts is the cause of the executive impairment in $\mathrm{AD}$. If so, then executive functions appear sustained by a distributed cortical neural network rather than by a unique frontal region and is very "sensitive" to disruption.

This view is supported by the results of the recent functional MRI study, which segregate different network components underlying the Wisconsin CST (WCST) ${ }^{39}$ The data revealed the entire neural network underlying WCST performance, including the right ventrolateral and dorsolateral prefrontal cortices, and the anterior cingulate cortex (ACC). Moreover, recent study of functional MRI and Stroop test offers concrete evidence of prefrontal circuit activation including the left medial frontal gyrus, left dorsolateral prefrontal cortex and bilaterally in the ACC. ${ }^{40}$ In addition, a study of schizophrenia with the WCST link poor performance at the test to reduced activation in the $\mathrm{ACC}_{i}^{41}$

A recent PET study by Mosconi et al. ${ }^{42}$ in MCI ApoE carriers subjects at higher risk of conversion to $\mathrm{AD}$ showed rCMRglc reduction within frontal areas, such as the $\mathrm{ACC}$ and inferior frontal cortex. Here, a symptomatic shift from MCI to AD may be related to the
Formatted

Formatted

Formatted

Formatted

Formatted

Formatted

Formatted

Formatted

Formatted
Formatted

Formatted

Formatted

Formatted

Deleted: anterior cingulate 
appearance of this frontal metabolic dysfunction, which could ensue from anterior-posterior cortico-cortical disconnection, which supports the hypothesis of early executive impairment. A supplemental explanation of the appearance of attentional deficits in AD comes from the damage to the nucleus basalis of Meynert, which provides the major cholinergic innervation to the cortex, and which is affected by early pathological changes, and so may be responsible for attentional impairments.

There are some limitations to the current study. We cannot be certain about our rate of diagnostic misclassification because of the lack of neuropathological confirmation of our clinical diagnoses. However, the detailed clinical and neuropsychological assessments, the use of neuro-imaging to clarify cerebral abnormalities and, finally, the use of a trained research team consisting of a neuropsychologist, geriatrician, and neurologist (also trained in brain imaging) reasonably limited the chance for diagnostic misclassification. In our attempt to select homogeneous populations as much as we could, we paid special attention not to include in our study patients with subcortical vascular lesions or depression. The small number of our groups may also be a limitation, because of the lack of power in statistical analysis. When checking our results in addition to the above described highly reliable results of the Stroop Test and the MCST, we find tendency for statistical significance only in TMT-B. However, even if the TMT-B results become significant in larger goups study, its findings will not contradict with our suggestion of predominantly $\underline{\text { selective attention and response inhibition deficit in MCI patients. }}$

In conclusion, our results show that executive functions are impaired in MCI patients. Executive functions deficits appear predominantly in tasks requiring resolution of competing response tendencies, cognitive flexibility and self-monitoring. These results suggest that MCI should be identified by using a more detailed procedure for the assessment of cognitive decline than the evaluation of memory alone. The possible relationship between impaired executive functions and incipient dementia in individuals with MCI, and the question of whether detecting executive deficits could improve 
prediction of the conversion from $\mathrm{MCI}$ to $\mathrm{AD}$, remains to be investigated in follow-up studies. 


\section{References:}

1. Petersen RC, Smith GE, Waring SC, et al. Mild cognitive impairment: clinical characterization and outcome. Arch Neurol 1999;56:303-308.

2. Petersen RC, Doody R, Kurz A, et al. Current concepts in mild cognitive impairment. Arch Neurol 2001;58:1985-1992.

3. Winblad B, Palmer K, Kivipelto M, et al. Mild cognitive impairment--beyond controversies, towards a consensus: report of the International Working Group on Mild Cognitive Impairment. J Intern Med 2004;256:240-246.

4. Collie A, Maruff P: The neuropsychology of preclinical Alzheimer's disease and mild cognitive impairment. Neurosci Biobehav Rev 2000;24:365-374.

5. Arnáiz E, Almkvist O, Ivnik RJ, et al. Mild cognitive impairment: a cross-national comparison $J N N P$ 2004;75:1275-1280.

6. Grundman M, Petersen RC, Ferris SH, et al. Mild cognitive impairment can be distinguished from Alzheimer disease and normal aging for clinical trials. Arch Neurol 2004;61:59-66.

7. Arnaiz E, Almkvist O. Neuropsychological features of mild cognitive impairment and preclinical Alzheimer's disease. Acta Neurol Scand 2003;179:34-41.

8. Anderson SW, Tranel D. Neuropsychological consequences of dysfunction in human dorsolateral prefrontal cortex. In: Boller F, Grafman J, eds. Handbook of Neuropsychology. Amsterdam: Elsevier, 2002;7:145-156.

9. Ready RE, Ott BR, Grace J, et al. Apathy and executive dysfunction in mild cognitive impairment and Alzheimer disease. Am J Geriatr Psychiatry 2003;11:222228. 
10. Perry RJ, Watson P, Hodges JR. The nature and staging of attention dysfunction in early (minimal and mild) Alzheimer's disease: relationship to episodic and semantic memory impairment. Neuropsychologia 2000;38:252-271.

11. Rapp MA, Reischies FM. Attention and executive control predict Alzheimer disease in late life: results from the Berlin Aging Study (BASE). Am J Geriatr Psychiatry $2005 ; 13: 134-141$.

12. Berg L. Clinical Dementia Rating (CDR). Psychopharmacol Bull 1988;24:637-639.

13. Yesavage JA, Brink TL, Rose TL, et al. Development and validation of a geriatric depression screening scale: a preliminary report. J Psychiatr Res 1983;17:37-49.

14. Folstein MF, Folstein SE, McHugh PR. Mini Mental State: a practical method for grading the cognitive state of patients for the clinicians. J Psychiatr Res $1975 ; 12: 189-198$.

15. De Rotrou J, Forette F, Hervy MP, et al. The Cognitive Efficiency Profile: description and validation in patients with Alzheimer's disease. Int J Geriatr Psychiatry 1991;6:501-509.

16. De Rotrou J, Traykov L, Rigaud AS, et al. Profil cognitif des plus ages. In: Forette F, Christen Y, Boller F, eds. Démence et longévité. Paris: FNG, 1998;155-172.

17. American Psychiatric Association. Diagnostic and statistical manual of mental disorders. Washington DC: American Psychiatric Association, 1994.

18. Petersen RC, Smith GE, Ivnik RJ, et al. Apolipoprotein E status as a predictor of the development of Alzheimer's disease in memory-impaired individuals. JAMA $1995 ; 273: 1274-1278$.

19. Wechsler D. Measurment of Adult Intelligence. Baltimore MD: Williams \& Wilkins, 1958. 
20. Milner B. Interhemispheric differences in the localisation of psychological processes in man. Br Med Bull 1971;27:272-277.

21. Buschke H. Cued recall in amnesia. J Clin Neuropsychol 1984;6:433-440.

22. Grober E, Buschke H, Crystal H, et al. Screening for dementia by memory testing. Neurology 1988;38:900-903.

23. Gauthier L, Dehaut F, Joanette Y. The Bells test. A quantitative and qualitative test for visual neglect. Int J Clin Neuropsychol 1989;11:49-54.

24. Reitan RM. Validity of the Trail Making Test as an indication of the organic damage. Percept Mot Skills 1958;8:271-276.

25. Nelson HE. A modified card sorting test sensitive to frontal defects. Cortex $1976 ; 12: 313-324$.

26. Stroop JR: Studies of interference in serial verbal reactions. J Exp Psychol 1935; $18: 643-662$.

27. Kaplan E, Goodglass H, Weintraub S. The Boston Naming Test. Boston: Veterans Administration Medical Center, 1978.

28. Lezak MD. Neuropsychological assessment. Oxford: Oxford University Press, 1995.

29. Morris JC, Heyman A, Mohs RC, et al. The Consortium to Establish a Registry for Alzheimer's Disease (CERAD). Part I. Clinical and neuropsychological assessment of Alzheimer's disease. Neurology 1989;39:1159-1165.

30. Grober E, Lipton RB, Hall C, et al. Memory impairment on free and cued selective reminding predicts dementia. Neurology 2000;54:827-832. 
31. Nagahama Y, Okina T, Suzuki N, et al. Factor structure of a modified version of the wisconsin card sorting test: an analysis of executive deficit in Alzheimer's disease and mild cognitive impairment. Dement Geriatr Cogn Disord 2003;16:103-112.

32. Perry RJ, Hodges JR. Fate of patients with questionable (very mild) Alzheimer's disease: longitudinal profiles of individual subjects' decline. Dement Geriatr Cogn Disord 2000;11: 342-349.

33. Guarch J, Marcos T, Salamero M, et al. Neuropsychological markers of dementia in patients with memory complaints. Int J Geriatr Psychiatry 2004;19:352-358.

34. Chen P, Ratcliff G, Belle SH, et al Patterns of cognitive decline in presymptomatic Alzheimer disease: a prospective community study. Arch Gen Psychiatry $2001 ; 58: 853-858$

35. Alexopoulos P, Grimmer T, Perneczky R, et al. Progression to dementia in clinical subtypes of mild cognitive impairment. Dement Geriatr Cogn Disord 2006;22:2734.

36. Yaffe K, Petersen RC, Lindquist K, et al. Subtype of Mild Cognitive Impairment and Progression to Dementia and Death. Dement Geriatr Cogn Disord 2006;22:312-319.

37. Dudas RB, Clague F, Thompson SA, et al. Episodic and semantic memory in mild cognitive impairment. Neuropsychologia 2005;43:1266-1276.

Formatted: Bullets and Numbering

Formatted

38. Murphy KJ, Rich JB, Troyer AK. Verbal fluency patterns in amnestic mild cognitive impairment are characteristic of Alzheimer's type dementia. J Int Neuropsychol Soc 2006;12:570-574.

39. Grober E, Dickson D, Sliwinski MJ, et al. Memory and mental status correlates of modified Braak staging. Neurobiol Aging 1999;20:573-579.

40. Collette F, Van der Linden M, Dabe P, et al. Contribution of lexico-semantic processes to verbal short-term memory tasks: a PET activation study. Memory 2001;9:249-259. 
41. Lie CH, Specht K, Marshall JC, et al. Using fMRI to decompose the neural processes underlying the Wisconsin Card Sorting Test. NeuroImage 2006;30:10381049

42. Yücel M, Pantelis C, Stuart GW, et al. Anterior cingulate activation during Stroop task performance: a PET to MRI coregistration study of individual patients with schizophrenia. Am J Psychiatry 2002;159:251-254

43. Videbech P, Ravnkilde B, Gammelgaard L, et al. The Danish PET/depression project: performance on Stroop's test linked to white matter lesions in the brain. Psychiatry Res 2004;130:117-30

44. Mosconi L, Perani D, Sorbi S, et al. MCI conversion to dementia and the APOE genotype: a prediction study with FDG-PET. Neurology 2004;63:2332-2340.
Formatted

Formatted: Bullets and Numbering

Formatted

Formatted

Formatted 
Table 1. Demographic characteristics of patients with MCI and normal controls

\begin{tabular}{lcccc}
\hline & NC & MCI & F & p \\
& $(\mathrm{n}=20)$ & $(\mathrm{n}=20)$ & & \\
\hline Gender W/M & $6 / 14$ & $4 / 16$ & & 0.47 \\
Age, years & $73.3(7.0)$ & $73.2(8.0)$ & 0.0004 & 0.983 \\
Education, years & $12.8(3.3)$ & $12.1(3.1)$ & 0.471 & 0.496 \\
MMSE & $29.5(0.5)$ & $28.95(1.1)$ & 4.430 & 0.042 \\
\hline
\end{tabular}

Values are means $(\mathrm{SD}) ; \mathrm{NC}=$ Normal controls; $\mathrm{MCI}=$ mild cognitive impairment; MMSE $=$ Mini Mental State Examination 
Table 2. The neuropsychological performance of the two groups and the results of ANOVA on each test

$$
\begin{array}{llll}
\text { NC } & \text { MCI } & \text { F } & \text { p }
\end{array}
$$

\begin{tabular}{lcccc}
\hline Memory & & & & \\
Digit Span & $6.0(1.3)$ & $5.9(1.0)$ & 0.019 & 0.8921 \\
Corsi Test & $4.9(0.9)$ & $4.6(1.0)$ & 0.691 & 0.4109 \\
FCSRT (free recall) & $30.9(5.7)$ & $21.1(4.9)$ & 34.051 & $<0.0001$ \\
FCSRT (total recall) & $47.0(0.9)$ & $41.2(4.6)$ & 30.494 & $<0.0001$ \\
FCSRT(free delayed recall) & $13.1(2.4)$ & $8.9(2.4)$ & 38.824 & $<0.0001$ \\
FCSRT(total delayed recall) & $15.9(0.3)$ & $15.0(1.0)$ & 12.068 & 0.0013 \\
FCSRT (recognition) & $15.95(0.2)$ & $15.6(0.7)$ & 4.774 & 0.0351 \\
Attention/Executive function & & & & \\
Trail Making Test A (time) & $54.4(13.2)$ & $61.1(21.0)$ & 1.475 & 0.2320 \\
Trail Making Test B (time) & $120.1(57.5)$ & $157.2(70.8)$ & 3.313 & 0.0766 \\
Bell test (time) & $142.4(41.2)$ & $151.5(50.2)$ & 0.386 & 0.5382 \\
Digit Symbol & $41.2(10.3)$ & $39.4(12.5)$ & 0.248 & 0.6215 \\
Stroop Test (interference) & $37.1(9.6)$ & $28.6(10.9)$ & 6.749 & 0.0133 \\
MCST (categories) & $5.9(0.4)$ & $5.6(0.7)$ & 2.974 & 0.0927 \\
MCST (perseverations) & $0.4(0.7)$ & $2.2(2.7)$ & 7.445 & 0.0096 \\
MCST (errors) & $1.7(2.2)$ & $5.2(4.9)$ & 8.321 & 0.0064 \\
Praxis & & & & \\
Copy designs & $13.7(0.8)$ & $13.6(1.0)$ & 0.123 & 0.7282 \\
Language & & & & \\
Boston Naming Test & $14.9(0.4)$ & $14.8(0.4)$ & 0.165 & 0.6867 \\
Verbal Fluency (animals) & $20.1(4.5)$ & $19.7(4.2)$ & 0.086 & 0.7714 \\
Verbal Fluency (letter F) & $13.4(4.0)$ & $11.6(4.1)$ & 1.832 & 0.1839 \\
\hline
\end{tabular}

Values are means (SD); FCSRT $=$ Free and Cued Selective Reminding Test; MCST $=$ Modified Card Sorting Test. 